\title{
The Osmotic Framework Adsorbed Solution Theory: Predicting Mixture Coadsorption in Flexible Nanoporous Materials $\dagger$
}

\author{
François-Xavier Coudert*
}

Predictive analytical methods are crucial tools in the design of adsorptive gas separation processes. While they are widely used for rigid nanoporous solids, there is a lack for flexible materials, including the very topical and promising dynamic metal-organic frameworks (MOFs), which display such eye-catching phenomena as gate opening and breathing. We present here the Osmotic Framework Adsorbed Solution Theory (OFAST), which predicts the evolution of structural transitions a nd selectivity upon adsorption of fluid mixtures in flexible nanoporous solids, using as sole input experimental pure components isotherms.

\section{Introduction}

Porous metal-organic frameworks (MOFs) are an exciting class of nanoporous materials, displaying an extremely large range of crystal structures and host-guest properties. Among their numerous prospective applications, such as gas storage, catalysis and sensing, adsorptive gas separation is gaining considerable attention. ${ }^{1}$ Indeed, compared to other classes of microporous materials currently used in such processes (e.g., zeolites, activated carbons, and silica gels), MOFs show a great potential due the wide possibilities of pre- or postsynthetic functionalization of their organic linkers. Moreover, a growing number of candidates for adsorptive separation processes are materials exhibiting a flexible porous framework, which respond to externally imposed control parameters (pressure, temperature, or adsorption of guest molecules) by changes in their structure. These systems have received much attention recently, and include such diverse phenomena as swelling (progressive, continuous deformation upon host adsorption or removal), ${ }^{2}$ gate opening (transition from a closed, nonporous phase to an open, microporous structure) ${ }^{3,4}$ and breathing (two successive structural transitions, $\mathbf{1} \rightarrow \mathbf{2} \rightarrow \mathbf{1}$ ). ${ }^{5}$

A large number of adsorptive gas separation processes are used today in industry, using adsorbents such as zeolites and activated carbons. The technical design of any adsorption process relies on information about the adsorption equilibria of multicomponent systems in a large number of different thermodynamic conditions, whose experimental determination is

$\dagger$ Electronic Supplementary Information (ESI) available: detailed mathematical derivations. See DOI: 10.1039/b000000x/

Chimie ParisTech and CNRS, 11 rue Pierre et Marie Curie, F-75005 Paris, France. Tel: +33(0)1 442767 51; E-mail: fx.coudert@chimie-paristech.fr

\$ Footnotes should appear here. These might include comments relevant to but not central to the matter under discussion, limited experimental and spectral data, and crystallographic data. expensive and time-consuming, considering the very large dimensionality of the parameter space for this problem. Thus, the industrial success of adsorptive separation processes, which rely on finding optimal conditions for gas separation in a given adsorbent, is linked to a great extent to the existence of a host of methods that predict multicomponent equilibrium properties based on pure component adsorption data. The simplest of these methods is the Ideal Adsorbed Solution Theory (IAST), ${ }^{6}$ but many more elaborate methods are used to take into account the nonideality of fluid mixtures. ${ }^{7}$

For flexible nanoporous materials, however, the well-known coadsorption models are not applicable, as they fail to take into account the guest-induced changes in the structure upon adsorption. There is thus a need for theoretical models allowing the prediction of structural transitions and coadsorption properties of mixtures in flexible nanoporous materials. This is exemplified by the severe lack of experimental data on gas coadsorption in flexible MOFs ${ }^{8,9}$, compared to the published data available for pure component adsorption. ${ }^{1}$

In this paper, we explain how to couple the standard adsorbed solution theories with thermodynamic equations of the osmotic ensemble (which describe the host phase equilibria) to predict coadsorption in flexible nanoporous materials, with pure component adsorption isotherms as the only input. We termed this family of theoretical methods OFAST, for Osmotic Framework Adsorbed Solution Theory. The simplest variant of OFAST, relying on IAST for prediction of coadsorption in each phase and Langmuir fits of the experimental isotherms, was very recently illustrated and showcased on archetypical systems exhibiting gate opening and breathing. ${ }^{10}$ Here, we present the generic derivation of the OFAST method from thermodynamic equations, the approximations made to obtain the simple "IAST+Langmuir" variant and the particular properties of its implementation. We then study the sensitivity of this method to the fits of the ex- 
perimental data. Finally, we present and validate a very simple, approximate analytical expression for the gate-opening pressure upon adsorption of mixtures.

\section{The OFAST method}

\subsection{Coadsorption in the osmotic ensemble}

The appropriate thermodynamic ensemble to describe the adsorption of fluids in a flexible material is the osmotic ensemble, ${ }^{11-15}$ where the control parameters are the number of molecules of the host framework, $N_{\text {host }}$, the mechanical constraint exerted on the system $\sigma$, the temperature $T$, and the chemical potentials of the adsorbed fluids, $\left\{\mu_{i}\right\}$. If we restrict the current study to materials with only a few metastable framework structures, it was demonstrated in earlier work that the use of an "osmotic subensemble" adequately describes the equilibrium between host structures upon adsorption. ${ }^{15,16}$ This is especially appropriate for materials that present abrupt phase transitions between well-defined frameworks structures, as opposed to phenomena like continuous swelling upon adsorption. The use of equations derived in the osmotic subensemble was validated on a large range of systems and phenomena, including such contrasting behaviors as the "breathing" of MIL-53 (Al) and the "gate opening" of $\mathrm{Cu}\left(4,4^{\prime}\right.$-bipy $)(\mathrm{dhbc})_{2}$. In this section, we present the thermodynamic framework to study the structural transitions induced in flexible nanoporous materials by the coadsorption of fluid mixtures. To this effect, we extend the framework developed for pure-component adsorption in ref. 16 and show what are the necessary assumptions to obtain reasonably simple equations linking mixture adsorption, host properties and structural transitions.

We consider the thermodynamic equations describing the adsorption of a mixture of fluids, $i \in\{\mathrm{A}, \mathrm{B}, \ldots\}$, inside a porous flexible material that is in equilibrium between a given number of metastable structures $k \in\{\alpha, \beta, \ldots\}$. Following the "osmotic subensemble" approach, to each metastable phase $k$ of host framework structure is associated a value of the osmotic potential restricted to that phase, $\Omega_{k}^{\text {os }}$. This potential depends on the control parameters of the ensemble: $\Omega_{k}^{\text {os }}=$ $\Omega_{k}^{\text {os }}\left(N_{\text {host }}, \sigma, T,\left\{\mu_{i}\right\}\right)$. It is equal to the sum of the Gibbs free energy of the isolated host structure in phase $k, G_{\text {host }}^{(k)}$, and the grand canonical potential for the adsorbate in the structure considered as rigid, $\Omega_{k}$.

In order to express the grand canonical potential as a function of quantities directly available from experiments or simulations, we calculate it from its derivatives, using the fundamental relation involving the chemical potential of the adsorbates: ${ }^{17-19}$

$$
\left(\frac{\partial \Omega}{\partial \mu_{i}}\right)_{V_{k}, T, \mu_{j \neq i}}=-N_{i}
$$

As we consider here the process of coadsorption of a mixture of fluids of fixed composition inside the porous material, the chemical potentials of the fluids are not at all independent. Indeed, if we note $\mathbf{y}=\left\{y_{\mathrm{A}}, y_{\mathrm{B}}, \ldots\right\}$ the molar composition of the fluid (such that $\sum y_{i}=1$ ), and $P$ the total external pressure of the gas mixture, then the chemical potentials are functions of $P$ and the composition: $\mu_{i}=\mu_{i}(P, \mathbf{y})$. In the example of an ideal mixture of ideal gases, this function would be:

$$
\mu_{i}(P, \mathbf{y})=\mu_{i}^{\circ}+R T \ln \left(\frac{y_{i} P}{P^{\circ}}\right)
$$

Thus, for the adsorption of a fluid mixture in the material, the variation of the grand potential with pressure is given by:

$$
\left(\frac{\partial \Omega}{\partial P}\right)_{V_{k}, T}=-\sum_{i} N_{i}\left(\frac{\partial \mu_{i}}{\partial P}\right)=-\sum_{i} N_{i} V_{\mathrm{m}, i}
$$

where $V_{\mathrm{m}, i}$ is the partial molar volume of fluid $i$ in the mixture. The osmotic grand potential restricted to phase $k$ can thus be written as: ${ }^{16}$

$$
\begin{aligned}
& \Omega_{k}^{\mathrm{os}}(T, P, \mathbf{y})=\Omega_{k}^{\mathrm{os}}(T, P=0, \mathbf{y})+\int_{0}^{P}\left(\frac{\partial \Omega}{\partial P}\right)_{V_{k}, T} \mathrm{~d} p \\
& =G_{k}^{\text {host }}(T)-\int_{0}^{P}\left(\sum_{i} N_{i}^{(k)}(T, p, \mathbf{y}) V_{\mathrm{m}, i}(T, p, \mathbf{y})\right) \mathrm{d} p \\
& =F_{k}^{\text {host }}(T)+P V_{k}-\int_{0}^{P}\left(\sum_{i} N_{i}^{(k)}(T, p, \mathbf{y}) V_{\mathrm{m}, i}(T, p, \mathbf{y})\right) \mathrm{d} p
\end{aligned}
$$

where $N_{i}^{(k)}(P)$ is the adsorbed quantity of fluid $i$ (i.e. the partial coadsorption isotherm) in the rigid framework structure of phase $k, V_{k}$ is the unit cell volume of the phase, and we equated the mechanical constraint $\sigma$ with the total external pressure $P$.

While eq. 4 is in theory enough to allow us to calculate the osmotic potential from properties of the mixture (partial molar volumes) and of the adsorption (isotherms), it can be significantly simplified in some commonly-encountered cases. As a first simplification, if the mixture of fluids is ideal, the molar volume is then independent of the mixture composition:

$$
\begin{aligned}
\Omega_{k}^{\mathrm{os}}(T, P, \mathbf{y}) & =F_{k}^{\text {host }}(T)+P V_{k} \\
& -\int_{0}^{P}\left(\sum_{i} N_{i}(T, p, \mathbf{y}) V_{\mathrm{m}, i}(T, p)\right) \mathrm{d} p
\end{aligned}
$$

Moreover, if the fluids themselves can be considered as ideal gases, then the equation simplifies even further to yield:

$$
\begin{aligned}
\Omega_{k}^{\text {os }}(T, P, \mathbf{y}) & =F_{k}^{\text {host }}(T)+P V_{k}-R T \int_{0}^{P} \frac{\sum_{i} N_{i}^{(k)}(T, p, \mathbf{y})}{p} \mathrm{~d} p \\
& =F_{k}^{\text {host }}(T)+P V_{k}-R T \int_{0}^{P} \frac{N_{\text {tot }}^{(k)}(T, p, \mathbf{y})}{p} \mathrm{~d} p
\end{aligned}
$$


where $N_{\text {tot }}^{(k)}$ is the total quantity of fluid adsorbed inside the pores of the material. In particular, in the very common case of a host material in equilibrium between two structures (and of an ideal mixture of ideal gases), the osmotic potential difference between the two phases follows a very simple equation:

$$
\begin{aligned}
& \Delta \Omega^{\mathrm{os}}(T, P, \mathbf{y}) \\
& =\Delta F^{\mathrm{host}}(T)+P \Delta V-R T \int_{0}^{P} \frac{\Delta N_{\mathrm{tot}}(T, p, \mathbf{y})}{p} \mathrm{~d} p
\end{aligned}
$$

\subsection{Predicting structural transitions upon adsorption of fluid mixtures}

In this section, we describe the general principles of the OFAST (Osmotic Framework Adsorbed Solution Theory) method to predict structural transitions upon adsorption of fluid mixtures in flexible nanoporous materials (schematized in Fig. 1). In particular, we show how all the terms needed in equations 4,5 and 6, which determine the relative stability of the host phases, can be derived from experimental, pure-component adsorption isotherms.

Using eq. 4 (or eq. 5 or 6 if the mixture or the fluids can be considered ideal), we can compute for a given composition of the fluid mixture and a given pressure the thermodynamic potential associated with each host phase. The most stable phase for each set of thermodynamic conditions is then the structure corresponding to the lowest osmotic potential, and the guest-induced structural transitions of the host upon coadsorption correspond to the pressures at which $\Omega_{k}^{\text {os }}(T, P, \mathbf{y})=\Omega_{k^{\prime}}^{\text {os }}(T, P, \mathbf{y})$. In the hypothesis that structural transitions happen near the thermodynamic equilibrium (i.e. that hysteresis loops are not too large), ${ }^{20}$ it is thus possible to predict and rationalize the behavior of flexible nanoporous materials upon adsorption of mixtures of fluid, based simply on properties of the mixture $-V_{\mathrm{m}, i}(T, p, \mathbf{y})-$ on properties of the empty host material $-F_{k}^{\text {host }}$ and $V_{k}-$ and on the partial adsorption isotherms of the mixture $-N_{i}^{(k)}(T, p, \mathbf{y})$. These few properties can be determined in a number of ways and, with the exception of the unit cell volumes $\left(V_{k}\right)$ which are known from the cristallographic structures of the material, they are not trivial to determine.

The determination of the relative free energies $\left(F_{k}\right)$ is a point of great difficulty. It was demonstrated in previous work on adsorption-induced structural transitions that they can be deduced from experimental isotherms and transition pressures, ${ }^{16}$ avoiding the uncertainties of an experimental determination by calorimetry ${ }^{21}$ or a theoretical prediction by first-principles simulations (which have difficulties calculating accurately entropies). The determination of relative free energies $F_{k}$, up to an additive constant, is made possible by the special case of eq. 4 for a pure component fluid:

$$
\Omega_{k}^{\mathrm{os}}(T, P)=G_{k}^{\text {host }}(T)-\int_{0}^{P} N^{(k)}(T, p) V_{\mathrm{m}}(T, p) \mathrm{d} p
$$

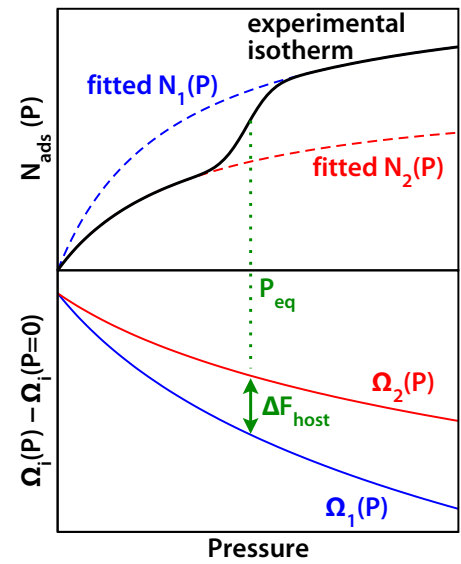

Fig. 2 Example of determination of the free energy difference $\Delta F_{\text {host }}$ between two phases of flexible material from fits of an experimental, stepped pure-component adsorption isotherm. ${ }^{16}$

Thus, if "rigid host" adsorption isotherms $N^{(k)}(P)$ for the pure component can be extrapolated as partial fits from an experimental stepped isotherm (as shown in Fig. 2, upper panel; each part of the stepped isotherm is fitted by a separate Langmuir equation, see Ref. 16 for details), the relative free energies of the host phases can be calculated from the structural transition pressures and integration of the fitted isotherms. This process is highlighted in Fig. 2.

Before fitting the experimental sorption isotherms, it should be noted that the thermodynamic quantities needed for the analysis are the total adsorbed amount (or "absolute quantities"), rather than the excess amount adsorbed that is typically obtained as a result of volumetric or gravimetric experiments. In particular, these two quantities will differ widely at high pressure, e.g. in the case of supercritical adsorption. They are linked by the following equation:

$$
N_{\text {abs }}=N_{\text {ex }}+\rho_{\text {bulk }} V_{\mathrm{p}}
$$

where $N_{\mathrm{abs}}$ is the absolute amount adsorbed, $N_{\mathrm{ex}}$ is the excess adsorption, $\rho$ is the fluid bulk density and $V_{\mathrm{p}}$ is the pore volume of the solid, which can come from experimental measurements of helium adsorption or from molecular simulation. ${ }^{22,23}$ This topic has been well explored, notably in order to link experimental excess data with the results of molecular simulations of adsorption, whose output is the absolute adsorption. In the present work, we used the experimental pore volumes reported for each structure studied.

Finally, the partial adsorption isotherms of the mixture $N_{i}^{(k)}(P)$ (or the total isotherm, $N_{\text {tot }}(P)$, in the case of ideal gases) can be determined using a variety of means. The direct measurement by an experimental setup for coadsorption, while possible, is known to be technically demanding. A di- 


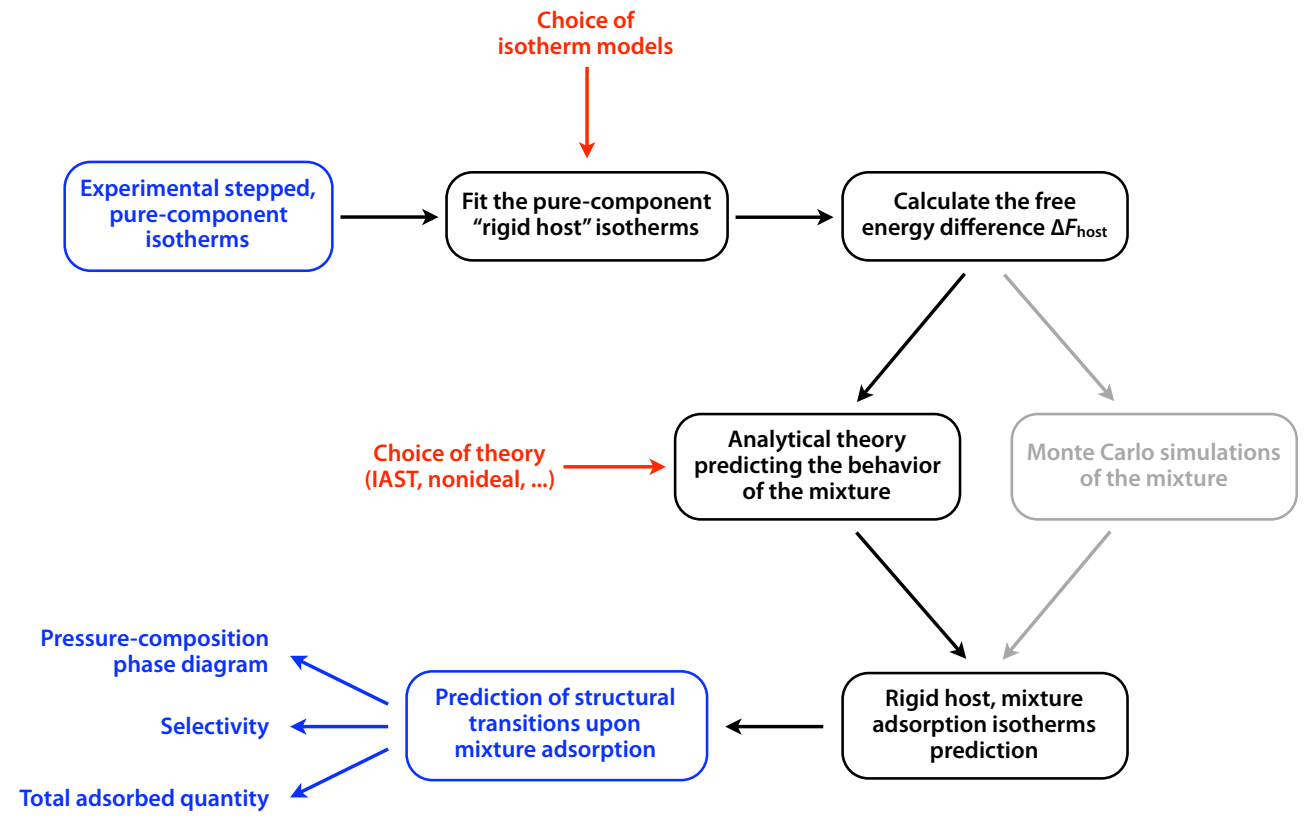

Fig. 1 Schematic description of the OFAST method for predicting the evolution of structural transition upon adsorption of fluid mixtures in flexible materials. In addition, the use of Monte Carlo simulations as an alternative to analytical theories for prediction of mixture coadsorption is indicated in gray.

rect determination by molecular simulation is possible and has been successfully applied in the past for related systems: ${ }^{24-26}$ the best tool for such a challenge is certainly the use of Grand Canonical Monte Carlo simulations coupled with forcefields validated on thermodynamics properties of the single-component adsorption of the various fluids considered. ${ }^{27}$ However, this still requires the exploration of a large parameter space, including mixture composition, pressure and the different phases of the host material. In order to perform a rapid prediction of coadsorption of mixtures in flexible materials, we propose here the use analytical models developped to predict coadsorption properties based on single-component quantities. These models include the Ideal Adsorbed Solution Theory (IAST) of Myers and Prausnitz, ${ }^{6}$ nonideal adsorbed solution models ${ }^{7,28}$ and the Vacancy Solution Theory (VST). ${ }^{29}$ While these models are no panacea for the prediction of mixture adsorption in microporous solids, they have been extensively studied in the past and both their areas of validity and their weaknesses have been assessed. In particular, they are known to be fairly reliable for adsorption of small gas molecules, or mixtures of apolar fluids of a similar chemical nature (such as mixtures of hydrocarbons).

In conclusion, by putting the methods described above together in a single scheme, we obtain the OFAST method. The method, outlined in fig. 1, relies on experimental, stepped purecomponent isotherms as an input. ${ }^{30}$ These isotherms are fitted to obtain "rigid host" pure-component isotherms and to cal- culate the free energy differences between phases. An analytical model is then used to obtain mixture coadsorption data in each host phase, and the osmotic subensemble equations then allow the prediction of structural transitions and a compositionpressure phase diagram for the flexible framework. The two main choices present in the method are the choice of isotherm models for fitting pure-component experimental data, and the choice of an analytical model for the prediction of coadsorption behavior. The most simple choices to implement the OFAST method is to use the Langmuir equation for pure-component isotherms, and the IAST for coadsorption prediction. More complicated alternatives could be considered, including nonideal models of fluid mixtures ${ }^{7,28}$ and other functional forms for the fitting of isotherms. Indeed, recent work on MOFs with dynamic frameworks by Wang et al. have successfully used the Langmuir-Freundlich equation in an extension of our group's earlier work on the osmotic ensemble. ${ }^{31}$ We show in the following that the particular combination "Langmuir+IAST" is numerically fast to solve, describes real-life systems in good agreement with available experimental data, and can even yield analytical solutions in the most simple cases. 


\section{Combining IAST and Langmuir pure- component isotherms}

In this section, we show how the generic OFAST method can be applied when combining Langmuir isotherms for fitting pure-component isotherms, and IAST for predicting mixture coadsorption. We first introduce notations and give a quick summary of the IAST equations, then combine them with Langmuir isotherms and determine the asymptotic behavior of their solution.

\subsection{The IAST equations}

We introduce here the IAST equations for the case of adsorption of a mixture of two fuilds $\mathrm{B}$ and $\mathrm{C}$ inside a nanoporous materials with fixed structure, based on known pure-component adsorption isotherms of $\mathrm{B}$ and $\mathrm{C}$ in the pores, noted as $N_{\mathrm{B}}^{*}(T, P)$ and $N_{\mathrm{C}}^{*}(T, P)$. We use the IAST to predict the thermodynamics of adsorption of a mixture of $\mathrm{B}$ and $\mathrm{C}$ inside the nanopores. The input quantities are the composition of the external fluid mixture, given by the molar fractions $y_{B}$ and $y_{C}=1-y_{B}$, and the total fluid pressure, $P$. The unknown quantities, which will be calculated by solving the IAST equations, are the composition of the adsorbed fluid, given by the adsorbed molar fractions $x_{\mathrm{B}}$ and $x_{\mathrm{C}}=1-x_{\mathrm{B}}$, and the total quantity of adsorbed fluid, $N_{\text {tot }}$. From these quantities, the values of other related properties can then be calculated, such as the adsorption selectivity, $\alpha=\frac{x_{\mathrm{B}} / x_{\mathrm{C}}}{y_{\mathrm{B}} / y_{\mathrm{C}}}$, and the adsorbed quantities of $\mathrm{B}$ and $\mathrm{C}, N_{\mathrm{B}}=x_{\mathrm{B}} N_{\text {tot }}$ and $N_{\mathrm{C}}=\left(1-x_{\mathrm{B}}\right) N_{\text {tot }}$.

In order to compute $x_{\mathrm{B}}$ and $N_{\text {tot }}$ for a certain value of $y_{\mathrm{B}}$ and $P$ (given $N_{\mathrm{B}}^{*}(T, P)$ and $N_{\mathrm{C}}^{*}(T, P)$ ), the IAST introduces the intermediate variables $P_{\mathrm{B}}^{*}$ and $P_{\mathrm{C}}^{*}$. There are then four IAST equations:

$$
\begin{gathered}
x_{\mathrm{B}}=\frac{P_{\mathrm{C}}^{*}-P}{P_{\mathrm{C}}^{*}-P_{\mathrm{B}}^{*}} \\
P y_{\mathrm{B}}=x_{\mathrm{B}} P_{\mathrm{B}}^{*} \\
\frac{1}{N_{\text {tot }}}=\frac{x_{\mathrm{B}}}{N_{\mathrm{B}}^{*}\left(P_{\mathrm{B}}^{*}\right)}+\frac{1-x_{\mathrm{B}}}{N_{\mathrm{C}}^{*}\left(P_{\mathrm{C}}^{*}\right)} \\
\int_{0}^{P_{\mathrm{B}}^{*}} N_{\mathrm{B}}^{*}(p) \mathrm{d} \ln p=\int_{0}^{P_{\mathrm{C}}^{*}} N_{\mathrm{C}}^{*}(p) \mathrm{d} \ln p
\end{gathered}
$$

This last equation can be rewritten as:

$$
\int_{0}^{P_{\mathrm{B}}^{*}} \frac{N_{\mathrm{B}}^{*}(p)}{p} \mathrm{~d} p=\int_{0}^{P_{\mathrm{C}}^{*}} \frac{N_{\mathrm{C}}^{*}(p)}{p} \mathrm{~d} p
$$

These four equations involve four unknowns $\left(x_{\mathrm{B}}, N_{\text {tot }}, P_{\mathrm{B}}^{*}\right.$ and $P_{\mathrm{C}}^{*}$ ). They can be solved numerically if the expressions of $N_{\mathrm{B}}^{*}(T, P)$ and $N_{\mathrm{C}}^{*}(T, P)$ are known, either as analytical fits or smoothed interpolation of the experimental data.

\subsection{IAST applied to Langmuir pure-component isotherms}

We now consider the adsorption of the same mixture of two gases $\mathrm{B}$ and $\mathrm{C}$, with the additional hypothesis that purecomponent adsorption of each fluid inside the porous material can be appropriately described by a Langmuir isotherm:

$$
N_{i}^{*}(P)=\frac{K_{i} P}{1+\frac{K_{i} P}{N_{i}}}
$$

where $K_{i}$ is the Henry constant for adsorption, which measures the affinity of the gas for the material, and $N_{i}$ is the maximum adsorbed quantity, i.e. the number of adsorbed gas molecules at the plateau of the isotherm. In that case, IAST equation 14 is as follows:

$$
N_{\mathrm{B}} \ln \left(1+\frac{K_{\mathrm{B}} P_{\mathrm{B}}^{*}}{N_{\mathrm{B}}}\right)=N_{\mathrm{C}} \ln \left(1+\frac{K_{\mathrm{C}} P_{\mathrm{C}}^{*}}{N_{\mathrm{C}}}\right)
$$

When combined with equations 10 and 11, it reduces to a single equation that determines $P_{\mathrm{B}}^{*}$ in terms of known quantities:

$$
\frac{P y_{\mathrm{C}} P_{\mathrm{B}}^{*}}{P_{\mathrm{B}}^{*}-P y_{\mathrm{B}}}=\frac{N_{\mathrm{C}}}{K_{\mathrm{C}}}\left[\left(1+\frac{K_{\mathrm{B}} P_{\mathrm{B}}^{*}}{N_{\mathrm{B}}}\right)^{\frac{N_{\mathrm{B}}}{N_{\mathrm{C}}}}-1\right]
$$

While equation 17 is analytical, there is no general expression for its solution in closed form. It is, however, much simpler to solve than the full system of IAST equations, especially since it does not involve numerical integration. In all cases presented in this article, we found that a simple Newton rootfinding algorithm with a starting value slightly larger than $P y_{\mathrm{B}}$ (typically, $P y_{\mathrm{B}}\left(1+10^{-3}\right)$ ) converges systematically. Because the OFAST method then needs to numerically integrate the coadsorption isotherms (eq. 7), both this steady convergence and the ability to solve the IAST equations in little computational time have allowed us to calculate a hundred points of a pressure-composition phase diagrams in a matter of minutes on a commodity PC with Mathematica. ${ }^{32}$

\subsection{Asymptotic behavior}

While the IAST equation describing the coadsorption of two fluids for which pure-component adsorption follows Langmuir isotherms (eq. 17) has no analytical solution, it is possible to establish the behavior of $P_{\mathrm{B}}^{*}(P)$ in the limits of low or high pressure, as a power series in $P$. All quantities of interest, including the selectivity $\alpha$ and the total adsorbed quantity $N_{\text {tot }}$ (of special interest because it is a crucial part of eq. 7), can thus be expressed as power series in $P$. For the sake of readability, the mathematical derivations of these power series is not given here but in Appendices A and B (see supporting information). We show here the (first order) asymptotic behavior of $\alpha$ and $N_{\text {tot }}$ because, to our knowledge, these expressions for the high-pressure asymptotes are not available in the existing literature. 


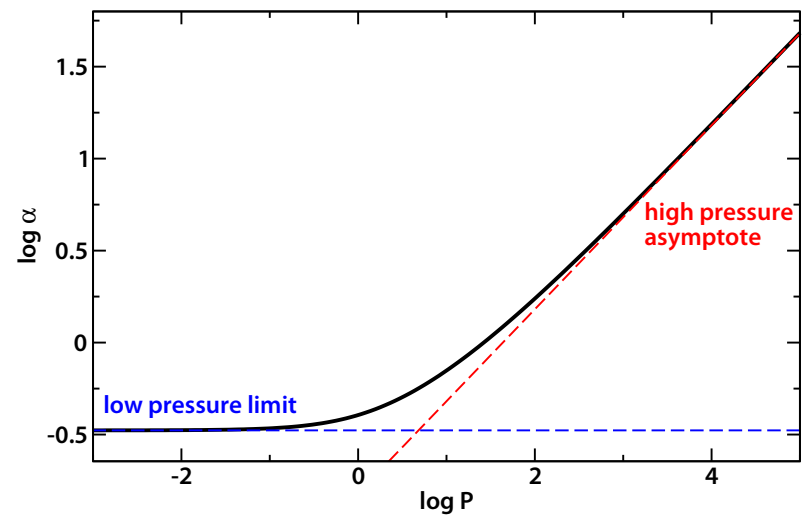

Fig. 3 An example of selectivity for a mixture of two fluids following Langmuir pure-component isotherms (solved numerically, in black), compared to the low-pressure (blue) and high-pressure (red) asymptotic behaviors established in the text.

At low pressure $(P \rightarrow 0)$, the first-order expressions are:

$$
\left\{\begin{array}{l}
\alpha=K_{\mathrm{B}} / K_{\mathrm{C}} \\
N_{\text {tot }}=P\left(y_{\mathrm{B}} K_{\mathrm{B}}+y_{\mathrm{C}} K_{\mathrm{C}}\right)
\end{array}\right.
$$

In the limit of high pressure $(P \rightarrow \infty)$, the first-order terms are (in the case where $N_{\mathrm{B}}>N_{\mathrm{C}}$ ):

$$
\left\{\begin{array}{l}
\alpha(P \rightarrow \infty) \simeq \frac{N_{\mathrm{C}}}{K_{\mathrm{C}}} \times\left(\frac{K_{\mathrm{B}}}{N_{\mathrm{B}}}\right)^{\frac{N_{\mathrm{B}}}{N_{\mathrm{C}}}} \times\left(P y_{\mathrm{B}}\right)^{\frac{N_{\mathrm{B}}}{N_{\mathrm{C}}}-1} \\
N_{\text {tot }}=N_{\mathrm{B}}\left[1-\left(\frac{N_{\mathrm{B}}-N_{\mathrm{C}}}{N_{\mathrm{C}}}\right)\left(\frac{y_{\mathrm{C}} K_{\mathrm{C}}}{N_{\mathrm{C}}}\right)\left(\frac{P y_{\mathrm{B}} K_{\mathrm{B}}}{N_{\mathrm{B}}}\right)^{-\frac{N_{\mathrm{B}}}{N_{\mathrm{C}}}}\right]
\end{array}\right.
$$

Figure 3 presents a numerical example of selectivity in a binary mixture of fluids that have single-component Langmuir-type adsorption, compared to the both the low-pressure and highpressure expressions. The high pressure asymptote can clearly be seen as a straight line (of slope $N_{\mathrm{B}} / N_{\mathrm{C}}-1$ ) in this log-log scale.

\section{Application to two archetypical flexible MOFs}

In this section, we apply the OFAST method as described above to two cases of flexible metal-organic framework. Well-characterized flexible porous MOFs where adsorption isotherms for more than one adsorbent has been recorded are scarce, and experimental data for mixture coadsorption are even rarer. Hence, we show here how the OFAST method applies to of two quite different phenomena observed in flexible MOFs: gate opening (or gated adsorption) in $\mathrm{Cu}\left(4,4^{\prime}\right.$-bipy $)(\mathrm{dhbc})_{2}$, and breathing (two successive structural transitions, in the form $1 \rightarrow 2 \rightarrow 1$ ) in MIL-53 (Al). While preliminary results on these

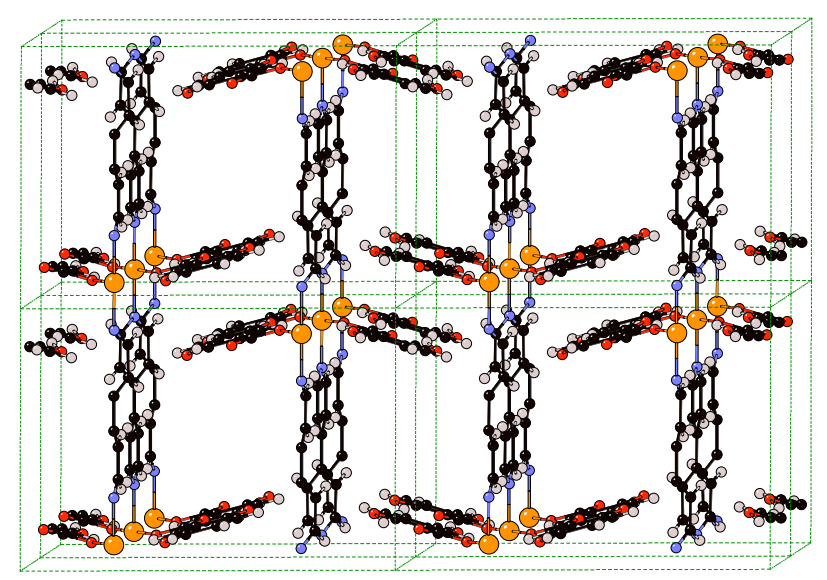

Fig. 4 Structure of the $\mathrm{Cu}\left(4,4^{\prime}\right.$-bipy $)(\mathrm{dhbc})_{2}$ coordination polymer shown as a $2 \times 2 \times 2$ supercell viewed along the $a$ axis. Atom colors are as follows: $\mathrm{Cu}$ - orange, $\mathrm{N}$ - blue, $\mathrm{C}$ - black, $\mathrm{O}$ - red, $\mathrm{H}$ - gray.

archetypical materials were reported in a previous study, ${ }^{10}$ we extend here these results to include approximate, simple analytical expressions for gate-opening and breathing pressures, an analysis of the sensitivity of the results to uncertainties in the parameters extracted from experimental data, and a discussion on the universality of the shape of the pressure-composition phase diagrams observed in the two specific cases reported here.

\subsection{Gate opening of $\mathrm{Cu}\left(4,4^{\prime}-\mathrm{bipy}\right)(\mathrm{dhbc})_{2}$}

A number of flexible hybrid frameworks present a structural transition from a nonporous structure to a porous one upon adsorption of guest molecules. This process is called gate opening (or gate adsorption) and has been reported in a number of compounds. ${ }^{3,33-35}$ These materials are expected to find applications as sensors, switches or actuators. Because the pressure at which the structural transition (referred to as "gate-opening pressure") happens generally depends on the guest, these materials are stated as having an important potential for gas separation. The reason for this is probably that the pure component adsorption isotherms lead to belief that in a mixture, one component would adsorb but the other would not. We show, on the example of $\mathrm{Cu}\left(4,4^{\prime}\right.$-bipy) $(\mathrm{dhbc})_{2}$, why this is not generally true and we predict how the gate-opening pressure for adsorption of a mixture evolves as a function of its composition.

$\mathrm{Cu}\left(4,4^{\prime}\right.$-bipy $)(\mathrm{dhbc})_{2} \cdot \mathrm{H}_{2} \mathrm{O}$ (4, $4^{\prime}$-bipy $=4,4^{\prime}$-bipyridine; dhbc $=2,5$-dihydroxybenzoate) was synthesized by Kitagawa et al. a few years ago ${ }^{3}$ and is known to exhibit a guest-induced structural transition upon adsorption of a large variety of gases $\left(\mathrm{CO}_{2}, \mathrm{O}_{2}, \mathrm{CH}_{4}\right.$ and $\left.\mathrm{N}_{2}\right)$ at $298 \mathrm{~K}$. The as-synthesized structure of this compound has been solved, and the crystal-to-crystal structural transition occurs between two phases hereafter labeled $\mathbf{1}$ 


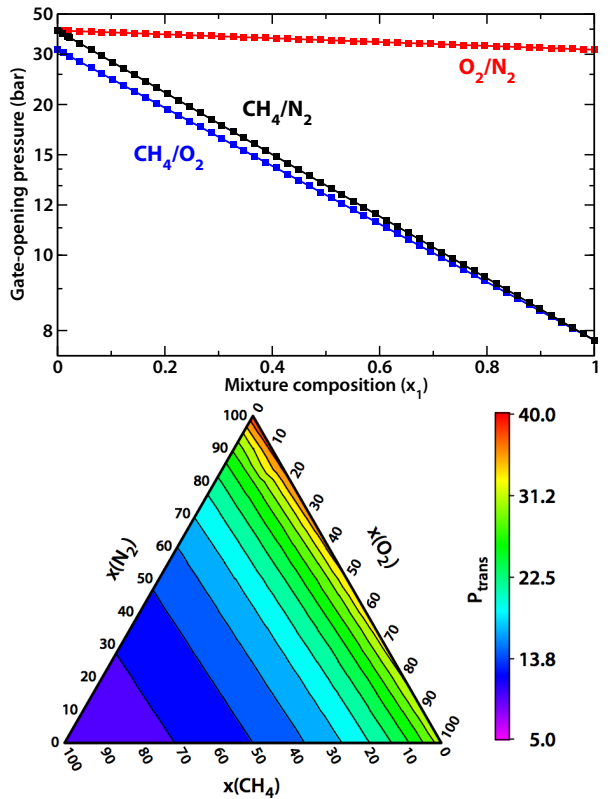

Fig. 5 Gate-opening pressure (in reciprocal scale) for adsorption of binary mixtures in $\mathrm{Cu}\left(4,4^{\prime} \text {-bipy) (dhbc }\right)_{2}$, as a function of mixture composition. Top panel: for $\mathrm{O}_{2} / \mathrm{N}_{2}$ (red), $\mathrm{CH}_{4} / \mathrm{N}_{2}$ (black), and $\mathrm{CH}_{4} / \mathrm{O}_{2}$ (blue) binary mixtures; symbols correspond to numerical solution of the equations, while lines show the analytical expression obtained: $1 / P_{\text {mixture }}=x_{1} / P_{1}+x_{2} / P_{2}$. Lower panel: for the $\mathrm{CH}_{4} / \mathrm{O}_{2} / \mathrm{N}_{2}$ ternary mixture. and 2, whose structures have not been solved. The dehydrated material (1) shows no microporosity and, upon gas adsorption, its channels open up at a given (guest-dependent) gate-opening pressure to yield a fully open structure (2). The framework structure of $\mathbf{2}$ is the same as that of the as-synthesized material, and is shown in Fig. 4.

In order to predict the behavior of $\mathrm{Cu}\left(4,4^{\prime}\right.$-bipy $)(\mathrm{dhbc})_{2}$ upon adsorption of mixtures of $\mathrm{O}_{2}, \mathrm{CH}_{4}$ and $\mathrm{N}_{2}$, we use Langmuir fits of the experimental adsorption isotherms for the pure components ${ }^{3}$ and the value of free energy difference between structures 1 and 2, which was calculated from the isotherms to be $4 \mathrm{~kJ} / \mathrm{mol}( \pm 0.5 \mathrm{~kJ} / \mathrm{mol})$ at $298 \mathrm{~K} .{ }^{16}$ Using the OFAST method with IAST and the Langmuir fits, we can numerically calculate the integral in Eq. 7 and determine the gate-opening pressure for each possible mixture, as well as adsorption selectivity as a function of composition and pressure. Fig. 5 shows the evolution of gate-opening pressure for the $\mathrm{N}_{2} / \mathrm{O}_{2}, \mathrm{~N}_{2} / \mathrm{CH}_{4}$ and $\mathrm{O}_{2} / \mathrm{CH}_{4}$ binary mixtures (top panel) as well as for a ternary mixture of the three gases (bottom panel). The gate-opening pressure varies smoothly and monotonically between the gateopening pressures for the respective pure components. It is also to be noted that, because the gate-opening pressure for $\mathrm{CH}_{4}$ (around 8 bar) is much lower than that of $\mathrm{O}_{2}$ and $\mathrm{N}_{2}$ (i.e. $P_{\mathrm{CH}_{4}} \ll P_{\mathrm{O}_{2}} \approx P_{\mathrm{N}_{2}}$ ), the main factor determining the transition pressure upon adsorption of the ternary mixture is the $\mathrm{CH}_{4}$ molar fraction, $x_{\mathrm{CH}_{4}}$.

Because the evolution of the gate-opening pressure with mixture composition is so smooth, we investigated the possibility to derive an analytical formula for it from the IAST/Langmuir equations. Noting that, for all adsorbents, the gate opening happens before the plateau of the isotherm is reached, we developed a Langmuir-type solution to the IAST equation, exact at low pressure to the second order in $P$ (see Appendix $\mathrm{C}$, in the supporting information). This approach allows to obtain a simple, approximate expression for the gate opening pressure of a mixture:

$$
P_{\text {gate }}(\mathbf{y})=\left(\sum_{i} \frac{y_{i}}{P_{\text {gate }, i}}\right)^{-1}
$$

where the sum over $i$ runs over all components of the mixture. In this approximation, the gate-opening pressure of the mixture is thus the weighted harmonic mean of the gate-opening pressures for its components, the weights being the molar fraction of the components. This approximation is plotted for all three binary mixtures as solid lines on the upper panel of Fig. 5, and can be compared to the the numerical solution of the full OFAST equations (the symbols). It can be seen that the agreement is excellent for all mixtures, showing the value of this approximation. Furthermore, the agreement is also excellent for the ternary mixture, though it is not represented here. This is especially noteworthy because the approximation proposed here for the gate-opening pressure is very simple, and can be used without 


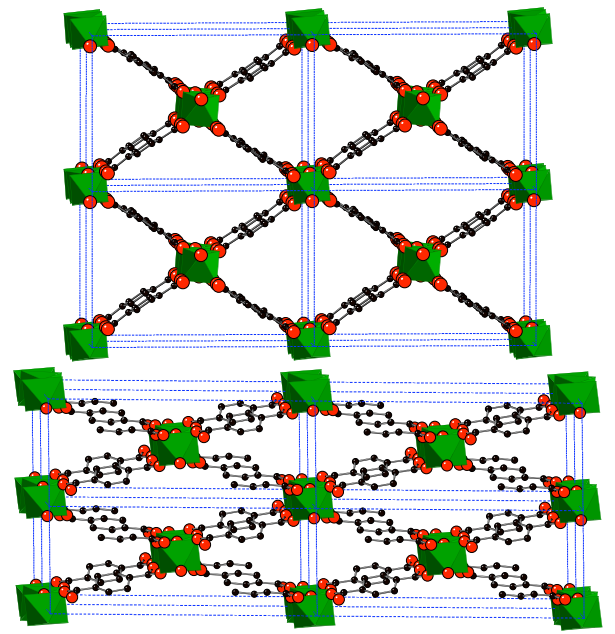

Fig. 6 View of the $2 \times 2 \times 2$ supercell of the $\mathbf{l p}$ (top) and $\mathbf{n p}$ (bottom) forms of MIL-53 (Al).

any calculation at all to predict roughly the gate-opening pressure for adsorption of mixtures, as long as it happens before the plateau of the isotherms.

\subsection{Breathing in MIL-53}

The MIL-53 metal-organic framework family ${ }^{36}$ has attracted a great deal of attention due to the massive flexibility it exhibits and the so-called "breathing" phenomenon, which consists of two successive guest-induced structural transition upon adsorption. The MIL-53 framework topology is formed of unidimensional chains of corner-sharing $\mathrm{MO}_{4}(\mathrm{OH})_{2}$ octahedra $\left(\mathrm{M}=\mathrm{Al}^{3+}\right.$, $\mathrm{Cr}^{3+}$ ) linked by 1,4-benzenedicarboxylate (BDC) ligands, which results in linear lozenge-shaped channels large enough to accommodate small guest molecules. This structure may oscillate between two distinct states, a large pore form (lp) and a narrow pore form (np; see Fig. 6), upon adsorption and desorption of gases. There is a $38 \%$ difference in cell volume between these two forms. The list of guests that induce this breathing includes $\mathrm{CO}_{2}, \mathrm{H}_{2} \mathrm{O}, \mathrm{C}_{n} \mathrm{H}_{2 n+2}(n \geq 2)$ and para-xylene, but neither $\mathrm{H}_{2}$, $\mathrm{CH}_{4}$, nor ortho- and meta-xylene at room temperature. ${ }^{5,37,38}$ This was shown to be due to the difference in adsorption affinity for the gas between the lp and $\mathbf{n p}$ phases. ${ }^{16}$ In the simplest case of Langmuir adsorption, it was further demonstrated that the difference in adsorption enthalpy $\Delta H_{\mathrm{ads}}=H_{\mathrm{ads}}^{(\mathbf{n p})}-H_{\mathrm{ads}}^{(\mathrm{lp})}$ conditions the occurence of breathing at a given temperature: if $\Delta H_{\text {ads }}$ is above a certain critical value, breathing takes places because gas adsorption favors strongly the $\mathbf{n p}$ phase at intermediate pressure; if $\Delta H_{\mathrm{ads}}$ is too small, breathing does not occur. ${ }^{39}$ Finally, at low enough temperature, the stable phase of the empty MIL-53 (Al) is the np phase ${ }^{40}$, and the breathing phenomenon is replaced by a single phase transition akin to gate opening. ${ }^{41}$
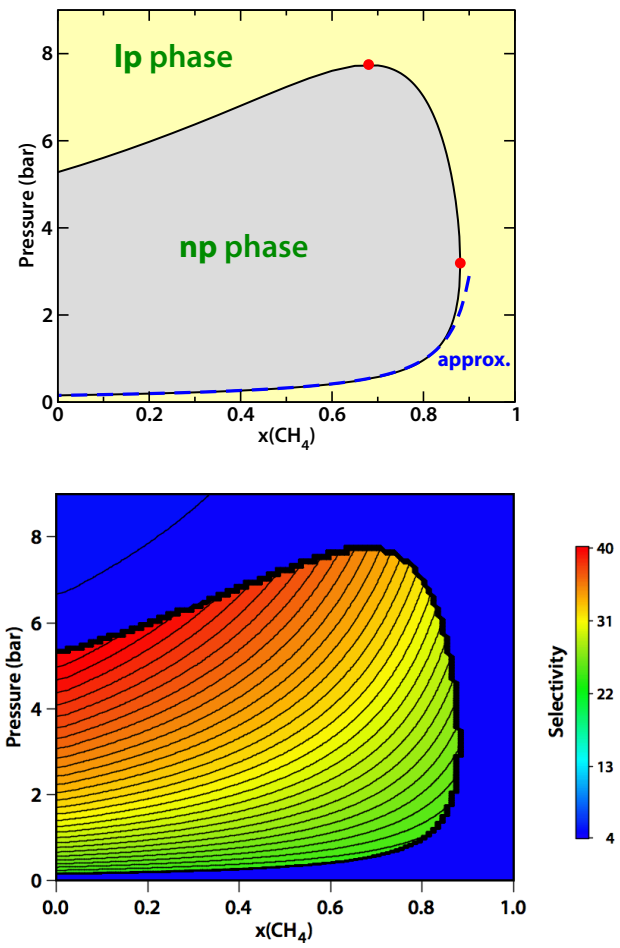

Fig. 7 Phase diagram of MIL-53 (Al) upon adsorption of a mixture of $\mathrm{CO}_{2}$ and $\mathrm{CH}_{4}$, as a function of pressure and mixture composition. Top: the dashed blue line shows the analytical approximation to the closing pressure. Bottom: $\mathrm{CO}_{2} / \mathrm{CH}_{4}$ selectivity is superimposed on the phase diagram.

Knowing that only some fluids induce breathing at a given temperature raises the question of what happens for a binary mixture of two components, one of which does trigger the two successive structural transition while the other does not. The first report of adsorptive gas separation using MIL-53 was published by Finsy et al., who studied $\mathrm{CH}_{4} / \mathrm{CO}_{2}$ separation using a fixed-bed of MIL-53 (Al) pellets. ${ }^{8}$ We applied the OFAST method to MIL-53 (Al), and showed that its prediction were consistent with the data collected by Finsy. ${ }^{10}$ Here, we briefly summarize these results before studying their dependence on all models parameters (such as parameters obtained while fitting the experimental isotherms) and discussing the generality of the features of the composition-pressure phase diagram obtained in the particular case of a $\mathrm{CH}_{4} / \mathrm{CO}_{2}$ mixture in MIL-53 (Al).

Relying on Langmuir fits of the experimental adsorption isotherms for $\mathrm{CO}_{2}$ and $\mathrm{CH}_{4}$ performed in ref. 39, and applying the OFAST method, we can calculate a composition-pressure phase diagram of MIL-53 (Al) upon adsorption of $\mathrm{CH}_{4}$ and $\mathrm{CO}_{2}$. This phase diagram, shown in Fig. 7, has many features worthy of note: firstly, there is a critical $\mathrm{CO}_{2}$ composition of around $12 \%$ below which no breathing occurs. Secondly, the evolution 


\begin{tabular}{lll}
\hline parameter & value & uncertainty \\
\hline$K_{\mathrm{CO}_{2}}^{\mathrm{p}}$ & $2.2 \mathrm{bar}^{-1}$ & $0.1 \mathrm{bar}^{-1}$ \\
$N_{\mathrm{CO}}^{\mathrm{lp}}$ & 10.1 & 0.1 \\
$K_{\mathrm{CO}_{2}}^{\mathrm{np}}$ & $11 \mathrm{bar}^{-1}$ & $\pm 2 \mathrm{bar}^{-1}$ \\
$N_{\mathrm{CO}_{2}}^{\mathrm{np}}$ & 2.7 & \pm 0.2 \\
$K_{\mathrm{CH}}^{\mathrm{lp}}$ & $0.55 \mathrm{bar}^{-1}$ & $\pm 0.01 \mathrm{bar}^{-1}$ \\
$N_{\mathrm{CH}}^{\mathrm{lp}}$ & 8.0 & \pm 0.2 \\
$K_{\mathrm{CH}}^{\mathrm{np}}$ & $0.55 \mathrm{bar}_{4}^{-1}$ & $\star$ \\
$N_{\mathrm{CH}_{4}}^{\mathrm{np}}$ & 2.1 & $*$ \\
\hline
\end{tabular}

Table 1 Uncertainties of the parameters fitted from experimental $\mathrm{CO}_{2}$ and $\mathrm{CH}_{4}$ isotherms in MIL-53 (Al). ${ }^{5,16,39}$ * indicates parameters that are not determined from experimental data, but derived from molecular simulation data. ${ }^{42}$

of the reopening transition (the higher-pressure $\mathbf{n p} \rightarrow \mathbf{l p}$ transition) with composition is nonmonotonical, and has a maximum around 8 bar. This maximal pressure is significantly higher than that of pure $\mathrm{CO}_{2}$, which is around 5 bar. Thirdly, and last, the $\mathrm{CO}_{2} / \mathrm{CH}_{4}$ adsorption selectivity predicted by IAST (Fig. 7, bottom panel) is much higher in the narrow-pore phase of the material than in its large-pore structure, as can be expected from the pure-component isotherms.

We now turn our attention to the sensitivity of the results to changes in the model parameters. The parameters fitted from experimental pure-component isotherms are reported in Table 1, along with estimated the uncertainty for each parameter. It can be seen that the parameters carrying the larger uncertainties are those pertaining to $\mathrm{CO}_{2}$ adsorption in the $\mathbf{n p}$ phase. The reason for this is that the low-pressure part of the experimental adsorption isotherm, which corresponds to the np phase and from which these two parameters are fitted, includes only a few disperse points. These uncertainties could be reduced if more detailed low-pressure data were available. We have also analyzed the uncertainty on $\Delta F_{\text {host }}$, the difference in free energy of the two empty phases of the material, which is a result of our model, calculated from the isotherm fits and the pressure at which structural transitions happen. This quantity, as a result, has two sources of uncertainty. The first is the uncertainties in the fitting parameters; it yields an uncertainty of roughly $\pm 10 \%$. The second is due to the fact that the equilibrium pressure of the structural transition, on which the model is based, can only be determined within a certain range, because of the presence of hysteresis in the experimental isotherms. Thus, the free energy difference can be located in a certain range, but may not be given a precise value if, for example, adsorption-desorption hysteresis is too broad. In the case of $\mathrm{CO}_{2}$ adsorption in MIL-53 (Al) at room temperature, we have further refined the value initially published $(2.5 \mathrm{~kJ} / \mathrm{mol})$ and propose here the more accurate range of $\Delta F_{\text {host }}=2.0 \mathrm{~kJ} / \mathrm{mol}( \pm 0.8 \mathrm{~kJ} / \mathrm{mol})$.

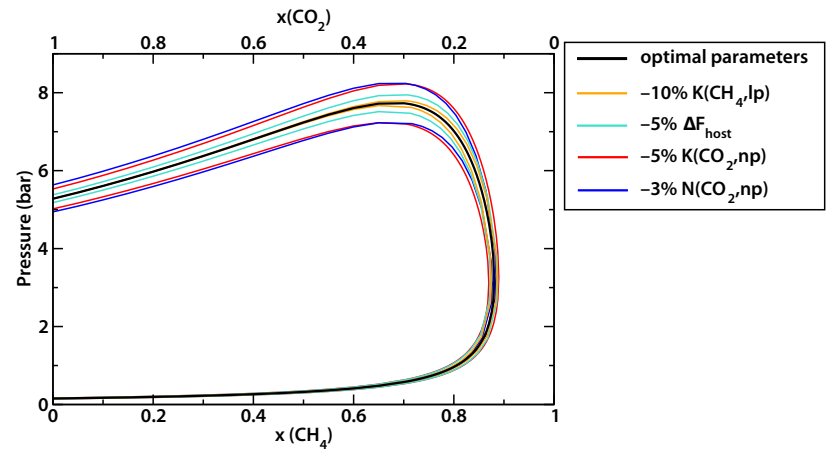

Fig. 8 Composition-presure phase diagrams of MIL-53 (Al) upon $\mathrm{CO}_{2} / \mathrm{CH}_{4}$ adsorption, with symmetric variations of the model parameters with the largest uncertainties.

Having now determined uncertainties of all parameters in the model, we can wonder what is their influence on the outcome of the OFAST method, i.e. on the pressure-composition phase diagram of MIL-53 (Al) and on the predicted coadsorption selectivities. We show in Figure 8 the effect of varying model parameters on this phase diagram; for reasons of clarity, only four parameters with the larger uncertainties are represented. It can be seen that, while the exact positions of phase transitions and particular points on the diagram are modified $\left(0.1 \leq x_{\lim } \leq 0.15\right.$, $7 \leq P_{\max } \leq 8$ bar), its shape and general features are preserved. In particular, the value of the critical $\mathrm{CO}_{2}$ composition required for breathing does not change much, and the nonmonotonical behavior of the reopening pressure as a function of composition is independent of the exact values of the model parameters. Similarly, we studied the influence of parameters to the $\mathrm{CO}_{2} / \mathrm{CH}_{4}$ selectivity. We showed that small variations of the parameters can lead to large changes in the values of selectivity (e.g., the selectivity range of the np phase can vary between 10-20 and 18-40), but the general behavior remains the same, with the narrow-pore structure having a much higher selectivity than the fully open structure.

We showed in previous work ${ }^{10}$ that the $\left\{\mathrm{CO}_{2} / \mathrm{CH}_{4}\right.$, MIL$53(\mathrm{Al})\}$ pressure-composition phase diagram resulting from OFAST predictions is consistent with the experimental results of Finsy et al. ${ }^{8}$ The results of this work were obtained by breakthrough experiments on a fixed-bed of MIL-53 (Al) pellets and, as such, do not directly represent the thermodynamic adsorption equilibrium. However, Hamon et $\mathrm{al}^{9}$ very recently published a study of adsorption of $\mathrm{CO}_{2} / \mathrm{CH}_{4}$ mixtures in the pores of MIL-53 (Cr). These newer results, in a material rather close to MIL-53 (Al) in structure and pure-component adsorption properties, include stepped adsorption isotherms and in situ Raman spectra. From the later, np/lp phase compositions were calculated as a function of mixture composition and vapour pressure. Both sets of data show that the evolution of closing 
and reopening pressure is in qualitative agreement with the predictions of OFAST. For example, the high-pressure reopening transition is seen to take place at increasing pressure $(8,9$ and 11 bars) for mixtures with an increasing composition in methane $\left(x_{\mathrm{CO}_{2}}=0,0.25\right.$ and 0.5 respectively). Similarly, the low-pressure closing transition pressure is seen to increase with the composition in methane (as seen from the curves of phase composition calculated from Raman results), although this effect is hard to quantify due to the large uncertainties of the data at low pressure. Moreover, there is a certain critical composition of the mixture for which the breathing effect disappears or is barely noticeable (even though the exact composition value differs, from $x_{\mathrm{CO}_{2}} \approx 0.12$ in the model to $x_{\mathrm{CO}_{2}} \approx 0.25$ in the experimental data). Thus, all the experimental results available to date are in qualitative agreement with the predictions of the OFAST method.

Finally, we present here an analysis of the $\left\{\mathrm{CO}_{2} / \mathrm{CH}_{4}, \mathrm{MIL}-\right.$ $53(\mathrm{Al})\}$ pressure-composition phase diagram based on approximate analytical expressions obtained in Section 3.3 and Appendix $\mathrm{C}$, with the aim of determining whether the shape and features of the phase diagram observed in this particular system can be expected to be more generic. We first focus on the low-pressure, $\mathbf{l} \mathbf{p} \rightarrow \mathbf{n p}$ closing transition. Since it happens at low pressure, before the plateau of the adsorption isotherms is reached, we can propose an analytical expression for the closing pressure that resembles Eq. 20:

$$
P_{\mathbf{l} \mathbf{p} \rightarrow \mathbf{n p}} \simeq \frac{x\left(\mathrm{CO}_{2}\right)}{P_{\mathbf{l p} \rightarrow \mathbf{n p}}^{\mathrm{CO}_{2}}}+\frac{x\left(\mathrm{CO}_{2}\right)}{P_{\mathbf{l p} \rightarrow \mathbf{n p}}^{\mathrm{CH}_{4}}}
$$

However, because $\mathrm{CH}_{4}$ does not induce breathing at room temperature, the term $P_{\mathbf{l p} \rightarrow \mathbf{n p}}^{\mathrm{CH}_{4}}$ is a negative, fictious transition pressure. In this case, by fitting the numericaly-obtained phase diagram, we determine its value to be $P_{\mathbf{l p} \rightarrow \mathbf{n p}}^{\mathrm{CH}_{4}} \simeq-3$ bar. The analytical approximation, which is displayed as a blue dashed line in Fig. 7, is in excellent agreement with the numerical transition pressure for compositions down to 0.15 . This shows, as in the case of gate opening, the very wide range of validity of this approximation. Based on this, we expect this part of the composition-pressure diagram of MIL-53 to be a generic feature, that would be applicable to other gas mixtures and other breathing materials.

In contrast, the analytical expression for the higher-pressure reopening $\mathbf{n p} \rightarrow \mathbf{l} \mathbf{p}$ transition is much more complicated. The existence of the nonmonotonical evolution of the reopening pressure $P_{\mathbf{n p} \rightarrow \mathbf{l}}$, and thus that of a maximum, is linked with the negative slope of $P_{\mathbf{n p} \rightarrow \mathbf{l}}\left(x_{\mathrm{CO}_{2}}\right)$ for $x_{\mathrm{CO}_{2}}$ close to 1 . It can be shown that the existence of this maximum is not a generic feature of the OFAST equations for breathing materials, but only exists when the equation below holds true:

$$
\begin{aligned}
& {\left[\left(N_{\mathrm{CO}_{2}}^{\mathbf{n p}}-N_{\mathrm{CH}_{4}}^{\mathbf{n p}}\right)\left(\frac{K_{\mathrm{CH}}^{\mathbf{n p}}}{N_{\mathrm{CH}_{4}}^{\mathbf{n p}}}\right)\left(\frac{K_{\mathrm{CO}_{2}}^{\mathbf{n p}}}{N_{\mathrm{CO}_{2}}^{\mathbf{n p}}}\right)^{-\frac{N_{\mathrm{CO}_{2}}^{\mathrm{np}}}{N_{\mathrm{CH}_{4}}^{\mathrm{np}}}}\right]} \\
& >\left[\left(N_{\mathrm{CO}_{2}}^{\mathbf{l p}}-N_{\mathrm{CH}_{4}}^{\mathbf{l p}}\right)\left(\frac{K_{\mathrm{CH}_{4}}^{\mathbf{l p}}}{N_{\mathrm{CH}_{4}}^{\mathbf{l p}}}\right)\left(\frac{K_{\mathrm{CO}_{2}}^{\mathbf{l p}}}{N_{\mathrm{CO}_{2}}^{\mathbf{l p}}}\right)^{-\frac{N_{\mathrm{CO}_{2}}^{\mathbf{l}}}{N_{\mathrm{CH}_{4}}^{\mathbf{p}}}}\right]
\end{aligned}
$$

The existence of this maximum relies on a balance between the adsorption affinities (enthalpic in nature) and the maximal adsorbed quantities (an entropic effect). It might not hold for some combinations of gases in MIL-53, or in other breathing materials, though we have not found to date a specific counterexample to present.

\section{Conclusion}

The coupling of adsorbed solution theories and the thermodynamic osmotic ensemble, composing the OFAST method, allows the prediction of coadsorption of mixtures in flexible nanoporous materials undergoing guest-induced phase transitions, with pure component adsorption isotherms as the only input. The quantities that can be calculated concern both the material (stability of each host phase with respect to total fluid pressure and composition) and the mixture (coadsorption selectivity, total adsorbed quantity). Herein, we derived the generic equations for OFAST, as well as the specific equations using Langmuir fits and the Ideal Adsorbed Solution Theory. We gave examples of applications of this method to binary and ternary gas mixture adsorbed in two archetypical flexible MOF, exhibiting phenomena called gate opening and breathing. We detailed some of the properties of the numerical resolution of the method, determined the low- and high-pressure asymptotic behavior of the analytical solutions, and tested the generality of the predicted pressure-composition phase diagrams and studied their sensitivity to parameters of the model. We derived a simple, approximate analytical expression for the gate-opening pressure of a mixture.

Finally, we would like to stress the fact that the OFAST method can be used with any choice of adsorbed solution theory, and any choice of isotherm model for fitting experimental data. As such, the quality of the results obtained are not limited to ideal solutions, but can be refined as much as the conventional methods for rigid hosts have been. We believe the OFAST method should prove crucial in designing adsorptive separation processes, by guiding the difficult and expensive experimental search for optimal materials and working conditions for gas separation. 


\section{Acknowledgments}

I thank Anne Boutin, Caroline Mellot-Draznieks and Alain Fuchs for discussions and insightful comments on this manuscript.

\section{Notes and references}

1 J.-R. Li, R. J. Kuppler and H.-C. Zhou, Chem. Soc. Rev., 2009, 38, 1477-1504.

2 C. Serre, C. Mellot-Draznieks, S. Surblé, N. Audebrand, Y. Filinchuk and G. Férey, Science, 2007, 315, 1828-1831.

3 R. Kitaura, K. Seki, G. Akiyama and S. Kitagawa, Angew. Chem. Int. Ed., 2003, 42, 428-431.

4 S. Kitagawa and K. Uemura, Chem. Soc. Rev., 2005, 34, 109-119.

5 S. Bourrelly, P. L. Llewellyn, C. Serre, F. Millange, T. Loiseau and G. Férey, J. Am. Chem. Soc., 2005, 127, 13519-13521.

6 A. L. Myers and J. M. Prausnitz, AIChE J., 1965, 11, 121-127.

7 R. T. Yang, Gas separation by adsorption processes, Imperial College Press, London, 1997.

8 V. Finsy, L. Ma, L. Alaerts, D. E. De Vos, G. V. Baron and J. F. M. Denayer, Microporous Mesoporous Mater., 2009, 120, 221-227.

9 L. Hamon, P. L. Llewellyn, T. Devic, A. Ghoufi, G. Clet, V. Guillerm, G. D. Pirngruber, G. Maurin, C. Serre, G. Driver, W. van Beek, E. Jolimaître, A. Vimont, M. Daturi and G. Férey, J. Am. Chem. Soc., 2009, 131, 1749017499.

10 F.-X. Coudert, C. Mellot-Draznieks, A. H. Fuchs and A. Boutin, J. Am. Chem. Soc., 2009, 131, 11329-11331

11 J. K. Brennan and W. G. Madden, Macromolecules, 2002, 35, 2827-2834.

12 B. J. Banaszak, R. Faller and J. J. de Pablo, J. Chem. Phys., 2004, 120, 1130411315.

13 R. Q. Snurr, A. T. Bell and D. N. Theodorou, J. Phys. Chem., 1994, 98, 51115119.

14 J. Shen and P. A. Monson, Mol. Phys., 2002, 100, 2031-2039.

15 M. Jeffroy, A. H. Fuchs and A. Boutin, Chem. Commun., 2008, 3275-3277.

16 F.-X. Coudert, M. Jeffroy, A. H. Fuchs, A. Boutin and C. Mellot-Draznieks, J. Am. Chem. Soc., 2008, 130, 14294-14302.

17 B. K. Peterson and K. E. Gubbins, Mol. Phys., 1987, 62, 215-226.

18 J. Puibasset and R. J. M. Pellenq, J. Chem. Phys., 2005, 122, 094704.

19 F. Cailliez, G. Stirnemann, A. Boutin, I. Demachy and A. H. Fuchs, J. Phys. Chem. C, 2008, 112, 10435-10445.

20 If the systems studied are far from thermodynamic equilibrium, other models need to be used. An example of one such model is reported in the recent work of Neimark et al. ${ }^{43}$.

21 A. Navrotsky, Phys. Chem. Miner., 1997, 24, 222-241.

22 A. V. Neimark and P. I. Ravikovitch, Langmuir, 1997, 13, 5148-5160.

23 A. L. Myers, J. A. Calles and G. Calleja, Adsorption, 1997, 3, 107-115.

24 H. Chen and D. S. Sholl, Langmuir, 2006, 22, 709-716.

25 D. Cao and J. Wu, Carbon, 2005, 43, 1364-1370.

26 R. Krishna and J. M. van Baten, Chem. Eng. J., 2007, 133, 121-131.

27 A. H. Fuchs and A. K. Cheetham, J. Phys. Chem. B, 2001, 105, 7375-7383.

28 M. B. Sweatman and N. Quirke, Langmuir, 2002, 18, 10443-10454.

29 S. Suwanayuen and R. P. Danner, AIChE J., 1980, 26, 68-76.

30 It should be stressed that not all stepped isotherms are caused by adsorptioninduced structural transitions of the host material. There are many examples of stepped adsorption isotherms in zeolites ${ }^{15,44}$ or $\mathrm{MOFs}^{45}$ that stem from structural rearrangements of the adsorbed phase.

31 Z. Wang and S. M. Cohen, J. Am. Chem. Soc., 2009, 131, 16675-16677.

32 Wolfram Research, Inc., Mathematica, Version 7.0, Champaign, IL (2008).

33 D. Li and K. Kaneko, Chem. Phys. Lett., 2001, 335, 50-56.
34 D. Tanaka, K. Nakagawa, M. Higuchi, S. Horike, Y. Kubota, T. C. Kobayashi, M. Takata and S. Kitagawa, Angew. Chem. Int. Ed., 2008, 47, 3914-3918.

35 A. J. Fletcher, K. M. Thomas and M. J. Rosseinsky, J. Solid State Chem., 2005, 178, 2491-2510.

36 C. Serre, F. Millange, C. Thouvenot, M. Nogues, G. Marsolier, D. Louër and G. Férey, J. Am. Chem. Soc., 2002, 124, 13519-13526.

37 G. Férey, M. Latroche, C. Serre, F. Millange, T. Loiseau and A. PercheronGuegan, Chem. Commun., 2003, 2976-2977.

38 L. Alaerts, M. Maes, L. Giebeler, P. A. Jacobs, J. A. Martens, J. F. M. Denayer, C. E. A. Kirschhock and D. E. De Vos, J. Am. Chem. Soc., 2008, 130, 14170 14178.

39 F.-X. Coudert, C. Mellot-Draznieks, A. H. Fuchs and A. Boutin, J. Am. Chem. Soc., 2009, 131, 3442-3443.

40 Y. Liu, J.-H. Her, A. Dailly, A. J. Ramirez-Cuesta, D. A. Neumann and C. M. Brown, J. Am. Chem. Soc., 2008, 130, 11813-11818.

41 A. Boutin, M.-A. Springuel-Huet, A. Nossov, A. Gédéon, T. Loiseau, C. Volkringer, G. Férey, F.-X. Coudert and A. H. Fuchs, Angew. Chem. Int. Ed., 2009, 48, 8314-8317.

42 P. L. Llewellyn, G. Maurin, T. Devic, S. Loera-Serna, N. Rosenbach, C. Serre, S. Bourrelly, P. Horcajada, Y. Filinchuk and G. Férey, J. Am. Chem. Soc., 2008, 130, 12808-12814.

43 A. V. Neimark, F.-X. Coudert, A. Boutin and A. H. Fuchs, J. Phys. Chem. Lett., 2010, 1, 445-449.

44 B. Smit and T. L. M. Maesen, Chem. Rev., 2008, 108, 4125-4184.

45 K. S. Walton, A. R. Millward, D. Dubbeldam, H. Frost, J. J. Low, O. M. Yaghi and R. Q. Snurr, J. Am. Chem. Soc., 2008, 130, 406-407. 